Carnets de géographes

GÉOGRAPHES.

\title{
Centralités et territorialités artistiques dans la structuration des espaces urbains
}

Le cas de Paris et Berlin

\section{Camille Boichot}

\section{(2) OpenEdition}

\section{Journals}

Édition électronique

URL : http://journals.openedition.org/cdg/872

DOI : $10.4000 /$ cdg. 872

ISSN : 2107-7266

\section{Éditeur}

UMR 245 - CESSMA

\section{Référence électronique}

Camille Boichot, "Centralités et territorialités artistiques dans la structuration des espaces urbains », Carnets de géographes [En ligne], 6 | 2013, mis en ligne le 01 septembre 2013, consulté le 24 septembre 2020. URL : http://journals.openedition.org/cdg/872 ; DOI : https://doi.org/10.4000/cdg. 872

\section{c) (†) $९$}

La revue Carnets de géographes est mise à disposition selon les termes de la Licence Creative Commons Attribution - Pas d'Utilisation Commerciale - Pas de Modification 4.0 International. 


\title{
CENTRALITES ET TERRITORIALITES ARTISTIQUES DANS LA STRUCTURATION DES ESPACES URBAINS
}

\author{
Le cas de Paris et Berlin
}

CAMILLE BOICHOT

La culture apparaît comme un élément de la définition du statut métropolitain d'une ville et comme un argument de distinction dans un contexte de compétition internationale entre les villes. La présence artistique dans les villes est souvent associée à des dynamiques de transformation et de revalorisation des espaces urbains. Cette thèse place au centre de l'analyse les espaces urbains de l'art contemporain dans deux métropoles artistiques majeures Paris et Berlin. Elle aborde de manière conjointe des enjeux liés au rayonnement culturel des deux métropoles et aux dynamiques urbaines liées à la présence artistique.

Cette thèse interroge les dynamiques urbaines qui sous-tendent l'organisation spatiale de l'art contemporain et cherche à comprendre dans quelle mesure l'organisation urbaine des espaces de l'art contribue à une spécialisation des espaces urbains métropolitains. Pour aborder ce questionnement général, deux hypothèses principales ont été testées dans ce travail : la première postule d'une organisation systémique et spécialisée des espaces de l'art et invite à considérer l'organisation urbaine de l'art contemporain à l'échelle métropolitaine, dépassant ainsi les études de cas localisées à un quartier ou cluster artistique et/ou créatif. La seconde pose que les circulations des artistes concourent à la structuration des espaces urbains à différentes échelles.

Pour tenter de valider ces hypothèses, une recherche comparative a été menée à l'échelle des métropoles parisienne et berlinoise, qui combine une analyse quantitative des lieux et des expositions de l'art contemporain et une analyse qualitative des pratiques et des discours d'artistes plasticiens installés à Montreuil et à Neukölln. Une base de données originale regroupant 407 lieux d'exposition et 1108 artistes exposés (janvier 2010) pour le cas berlinois et 338 lieux et 769 artistes exposés (janvier 2010) pour le cas parisien a permis, sur la base d'analyses statistiques multivariées, de caractériser l'organisation des lieux de l'art contemporain à Paris et à Berlin en termes de spécialisation fonctionnelle, de localisation ou encore de durée d'installation, ainsi que les dynamiques d'échanges artistiques qui qualifient la position de ces deux villes dans un système artistique international. L'analyse des pratiques spatiales des artistes à l'échelle intra-urbaine a d'autre part été menée sur la base d'entretiens, d'observation ethnographique et de participation observante dans deux espaces à forte présence artistique : Montreuil à Paris et Neukölln à Berlin. L'enquête qualitative répond au double objectif de comprendre les logiques territorialisantes de la création artistique et de saisir les articulations entre ancrages et circulations qui structurent les espaces de vie des artistes.

L'organisation des espaces urbains de l'art à Paris et à Berlin montre dans un premier temps malgré des différences qui tiennent à des histoires et des contextes urbains particuliers - l'existence de structures métropolitaines multipolaires caractérisées par des centralités spécialisées. Ces spécialisations tiennent d'une part à la nature même de l'activité artistique des lieux d'exposition, et d'autre part aux échanges artistiques observés au prisme des expositions d'art contemporain organisées à Paris et à Berlin et qui contribuent à souligner l'insertion de ces deux métropoles dans les réseaux artistiques globaux. Cette thèse donne donc à voir dans un premier temps des systèmes 
artistiques parisiens et berlinois où coexistent une dynamique d'échanges endogène forte et une animation de réseaux artistiques majeurs à différentes échelles soulignant ainsi le rôle de pivots de Paris et Berlin, à l'interface de réseaux artistiques locaux et globaux.

Les pratiques spatiales des artistes sont interrogées à l'échelle locale d'espaces urbains marqués par la création, à Montreuil dans le cas parisien et à Neukölln dans le cas de Berlin. Cette thèse montre, dans un second temps, comment ces pratiques sont déterminantes à la fois dans la définition d'espaces de création et dans les processus à l'œuvre dans l'affirmation collective d'une identité créatrice. Les processus de valorisation et a fortiori de gentrification urbaine auxquels contribuent les pratiques des artistes sont analysés finement et la comparaison des contextes urbains à Montreuil et Neukölln met en évidence la diversité des acteurs et des stratégies qui conduisent à la valorisation symbolique et immobilière de quartiers marqués par la présence des artistes.

En portant une attention particulière aux circulations des artistes, cette thèse montre enfin que la portée variable des circulations individuelles influe sur une structuration des réseaux à des échelles différentes : les circulations nombreuses à l'échelle de la ville soulignent le rôle des pratiques individuelles dans la définition et les dynamiques des réseaux artistiques intra-métropolitains. Dans un effort d'articulation entre ancrages et circulations, cette thèse explicite également le fonctionnement de territoires transnationaux au sein des métropoles.

À travers une approche qui croise les contextes urbains, les échelles d'analyse et les méthodes, cette thèse montre le rôle des espaces de l'art dans la fabrique de l'urbain, contribuant au renforcement des centralités métropolitaines existantes et aussi à l'émergence de centralités artistiques plus spécifiques et spécialisées.

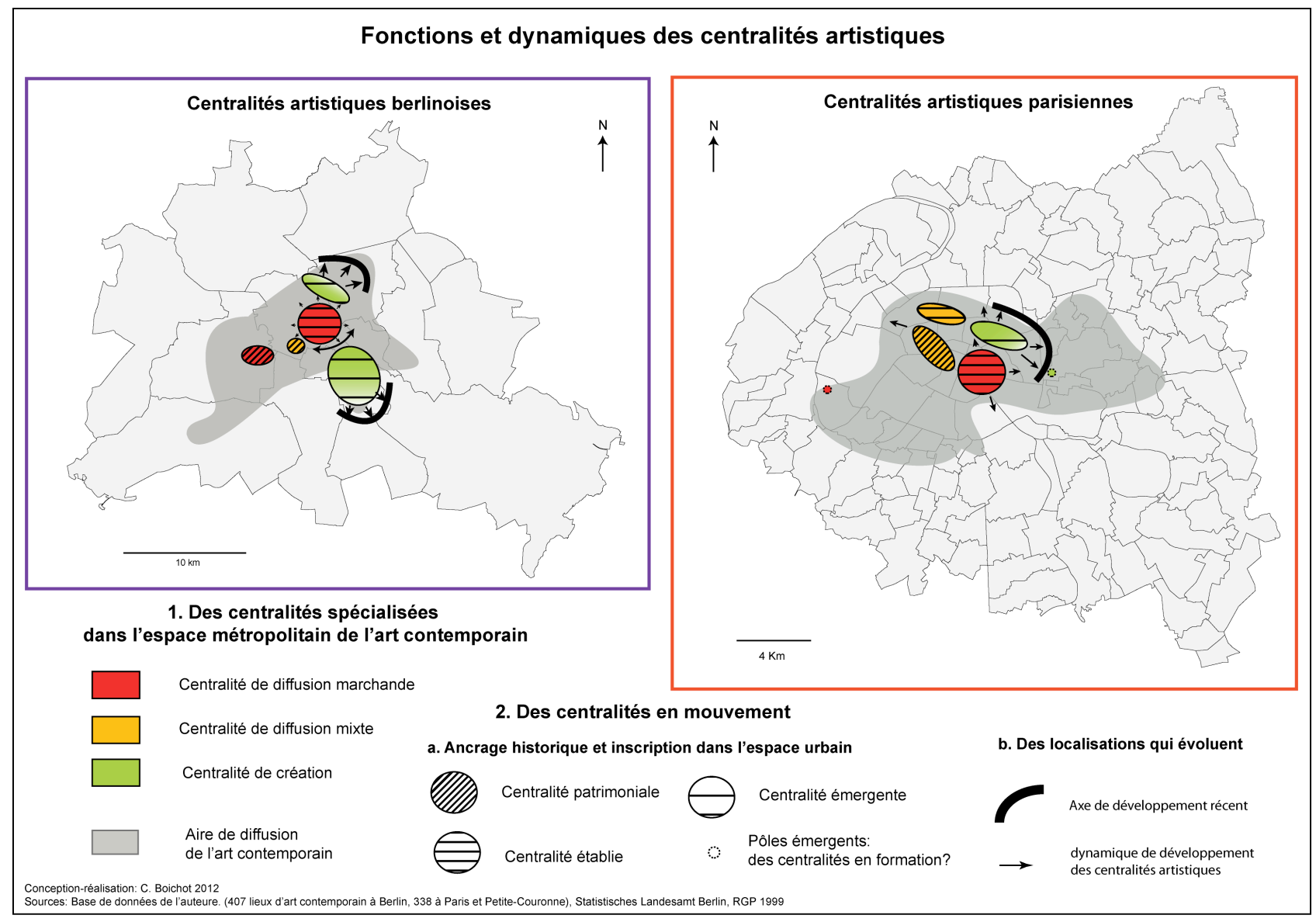


Fiche informative

Discipline

Géographie

Directeurs

Nadine Cattan (CNRS, UMR Géographie-Cités) et Prof. Dr. Stefan Krätke (Université Européenne Viadrina)

\section{Université}

Université Paris 1 Panthéon-Sorbonne et Université Européenne Viadrina

Membres du jury de thèse, soutenue le 12/12/2012

- Nadine Cattan, Directrice de recherche au CNRS

- Jean-Michel DECROLY, Professeur de Géographie et de Tourisme, Université Libre de Bruxelles, rapporteur

- Guy DI MÉO, Professeur de géographie, Université Michel de Montaigne, Bordeaux III, président

- Boris GRÉSILLON, Professeur de géographie, Université d'Aix-Marseille, rapporteur

- Prof. Dr. Stefan KRÄTKE, Professeur de géographie économique et sociale, Université Européenne Viadrina (Francfort sur l'Oder)

Situation professionnelle actuelle

Post-doctorat Université Libre de Bruxelles

\section{Courriel de l'auteur}

camille.boichot@gmail.com 